Folglich stellt sich die Frage, ob der Osten, wie Adam Pzreworski 1991 prophezeite, zum Süden anstelle zum Westen geworden ist ${ }^{45}$ und / oder nicht zum Trendsetter auch für die etablierten Demokratien. Dabei bieten die baltischen Republiken drei verschiedene Modelle: In Estland gibt es einen Dualismus zwischen den Parteien; die liberale Reformpartei und die konservative Vaterlandsunion / Res Publica werden von jenem Teil der Wählerschaft unterstützt, die zum Weg der vergangenen 20 Jahre keine Alternative sehen und davon weitgehend auch profitiert haben. Daneben existiert die Reformpartei als starke politische Kraft, die das russische Elektorat integriert und auch die wirtschaftlich weniger erfolgreiche Wählerschaft anspricht. In Litauen hingegen genießen die aus den Kommunisten hervorgegangenen Sozialdemokraten ebenso wenig Ansehen wie die aus der Regenschirm-Bewegung entstandene Vaterlandsunion, die sich an der Regierung abwechseln. Neue politische Kräfte waren wenig nachhaltig. In Lettland hingegen sind die dauernden Veränderungen im Parteiensystem so umfangreich, dass ein Überblick schwerfällt. Und da es Parteienkoalitionen erlaubt ist, mit gemeinsamen Listen zu kandidieren, ist auch kein Ende dieses Wandlungsprozesses abzusehen. Die Wahlen zur 9. Saeima 2006 haben gesetzeswidrig stattgefunden, wie das Verfassungsgericht 2008 festgestellt hatte, weil mit der Volkspartei und Lettlands Erster Partei / Lettlands Weg gleich zwei Listen gegen die Deckelung der Wahlkampfausgaben verstoßen hatten. Mehr als zwei Jahre nach dem Urnengang führte dies jedoch nicht zu sofortigen Neuwahlen. Angesichts der eingetretenen Krise und der Massenproteste im Lande wurde eher über die in der Verfassung vorgesehene Möglichkeit spekuliert, das Parlament auf Anregung des Präsidenten und mit anschließendem Referendum aufzulösen. Dies geschah aber auch nicht, sondern die reguläre Neuwahl am 2. Oktober 2010 läutete die nächste Runde der Wahlen im Baltikum ein.

45 Vgl. Adam Przeworski, Democracy and the Market: Political and Economic Reforms in Eastern Europe and Latin America, Cambridge 1991.

\title{
Die schwedische Reichstagswahl vom 19. September 2010 - Zur Logik einer sich auflösenden sozialdemokratischen Hochburg
}

\author{
Sven Jochem
}

Am 19. September 2010 wählten die schwedischen Wahlbürger einen neuen Reichstag. Nur geringe Verschiebungen der Stimmenrelationen im Vergleich zu 2006 führten zu großen Konsequenzen: Erstmals konnten die rechtspopulistischen Schwedendemokraten (SD) ins Parlament einziehen. Aber auch erstmals in der Nachkriegsgeschichte wurde eine bürgerliche Koalition an der Wahlurne bestätigt. Und noch nie seit 1920 erreichte die Sozialdemokratische Arbeiterpartei Schwedens (SAP) mit 30,7 Prozent der Stimmen ein schlechteres Wahlergebnis. Am 14. November 2010 trat die Parteivorsitzende und sozialdemokratische Spitzenkandidatin Mona Sahlin von ihren Ämtern zurück und entfachte eine lebhafte Personaldiskussion. 
Die Reichstagswahl 2010 steht für eine vorläufige Zuspitzung der Geschichte einer sich auflösenden sozialdemokratischen Hochburg in Europa. Diese Auflösung weist zum Teil Ähnlichkeiten zu Diversifizierungsprozessen in anderen europäischen Parteiensystemen auf $^{1}$; zum anderen Teil kann der Niedergang der schwedischen Sozialdemokratie auf strategische Unwägbarkeiten sowie - vor allem - auf das Geschick der Konservativen Partei während der globalen Finanzkrise zurückgeführt werden. In der Vergangenheit konnte eine hegemoniale Sozialdemokratie die Logik eines heterogenen bürgerlichen Lagers für ihre Zwecke nutzen - nun ist die Sozialdemokratie eingezwängt zwischen einem dominanten und intern kohäsiven bürgerlichen Block mit einer strategisch umsichtig agierenden Konservativen Partei einerseits sowie nur schwerlich zu überbrückenden programmatischen Differenzen im linken Parteienspektrum andererseits.

\section{Das Wablergebnis}

Die Reichstagswahl 2010 kann kaum als Erdrutschwahl bezeichnet werden (vgl. Tabelle 1). Die bürgerliche „Allianz“, bestehend aus Konservativer Partei (M), Zentrumspartei (C), Liberaler Volkspartei (FP) sowie Christdemokratischer Partei (KDS) konnte sich deutlich gegen das rot-grüne Wahlbündnis aus Sozialdemokratischer Arbeiterpartei (SAP), Grüner Partei (MP) sowie Linkspartei (V) durchsetzen. Nur knapp verfehlte die bürgerliche Parteienallianz die absolute Mehrheit der Sitze, da die rechtspopulistischen Schwedendemokraten (SD) die Vier-Prozent-Hürde deutlich überspringen und erstmals in den Reichstag einziehen konnten. Lange Zeit bestand für die bürgerlichen Parteien noch die Hoffnung, dass sich durch die verzögerte Auszählung der Stimmen ${ }^{2}$ zwei zusätzliche Mandate ergeben könnten, die ihre parlamentarische Mehrheit hätten knapp sichern können. ${ }^{3}$

Die Gewinner der Wahl waren neben den Schwedendemokraten die Grüne sowie die Konservative Partei. ${ }^{4}$ Während letztere im Vergleich zur Wahl 2006 3,9 Prozentpunkte zu-

1 Vgl. Jim Cronin / George Ross / James Shoch (Hrsg.), What's Left of the Left? Liberalism and Social Democracy in a Globalized World, Durham (im Erscheinen); Sven Jochem, Jenseits „Dritter Wege": Die Sozialdemokratie im Parteienwettbewerb - ein europäischer Vergleich, in: der moderne staat, 3. Jg. (2010), H. 1, S. 125 - 146.

2 In Schweden besteht für die Wahlbürger die Möglichkeit einer verfrühten Stimmabgabe. Hierbei können an bestimmten Orten, wie öffentlichen Bibliotheken, die Stimmen bereits vor dem offiziellen Wahltermin abgegeben werden. Die Auszählung dieser Stimmen zog sich über den Wahlabend hinaus hin und sorgte für zunächst unklare Mehrheitsverhältnisse in den Hochrechnungen.

3 Hinter der Differenz von zwei Mandaten verbergen sich lediglich knapp 1.800 Wählerstimmen, die zur Erreichung einer absoluten parlamentarischen Mehrheit für die Allianz notwendig gewesen wären. Es handelte sich also um ein äußerst knappes Wahlergebnis, das durch die Umrechnungsmodalitäten in Mandate deutlicher ausfällt, als dies tatsächlich der Fall ist. Vgl. das amtliche Endergebnis der schwedischen Wahlbehörde, http://www.val.se/val/val2010/slutresultat/ protokoll/protokoll_00R.pdf (Abruf am 10. November 2010).

4 In Schweden wurden zeitgleich auch die Wahlen zu den kommunalen und regionalen Parlamenten durchgeführt. Bei diesen Wahlen konnte die SAP besser abschneiden. Rechnet man die Ergebnisse aller Kommunalwahlen zusammen, dann kommt die SAP auf 32,4 Prozent, die Konservative Partei hingegen nur auf 26,2 Prozent. Vgl. Fredrik Gunér, S starkare i kommuner och landsting (staatliches Fernsehen), http://svt.se/2.128339/1.2159661/s_starkare_i_kommuner_ och_landsting (Abruf am 23. September 2010). 


\begin{tabular}{|c|c|c|c|c|}
\hline \multicolumn{5}{|c|}{ Tabelle 1: Ergebnisse der schwedischen Reichstagswabl 2010 und 2006} \\
\hline & \multicolumn{2}{|c|}{2010} & \multicolumn{2}{|c|}{2006} \\
\hline Partei & $\begin{array}{c}\text { Stimmen } \\
\text { (in Prozent) }\end{array}$ & Sitze & $\begin{array}{c}\text { Stimmen } \\
\text { (in Prozent) }\end{array}$ & Sitze \\
\hline Allianz & 49,4 & 173 & 48,2 & 178 \\
\hline M & 30,1 & 107 & 26,2 & 97 \\
\hline $\mathrm{FP}$ & 7,1 & 24 & 7,5 & 28 \\
\hline $\mathrm{C}$ & 6,6 & 23 & 7,9 & 29 \\
\hline $\mathrm{KD}$ & 5,6 & 19 & 6,6 & 24 \\
\hline Die Rotgrünen & 43,6 & 156 & 41,6 & 171 \\
\hline SAP & 30,7 & 112 & 35,0 & 130 \\
\hline MP & 7,3 & 25 & 5,2 & 19 \\
\hline V & 5,6 & 19 & 5,9 & 22 \\
\hline SD & 5,7 & 20 & 2,9 & - \\
\hline Übrige (Total) & 1,4 & - & 2,8 & - \\
\hline davon FI & 0,4 & - & 0,7 & - \\
\hline davon PP & 0,7 & - & 0,6 & - \\
\hline $\begin{array}{l}\text { Wahlbeteiligung } \\
\text { (in Prozent) }\end{array}$ & & & & \\
\hline \multicolumn{5}{|c|}{$\begin{array}{l}\text { Anmerkungen: Allianz: M = Moderata Samlingspartiet (Konservative Partei), FP = Folkpartiet Liberalerna } \\
\text { (Liberale Partei). KD = Kristdemokraterna (Christdemokratische Partei), C = Centerpartiet (Zentrums- } \\
\text { partei). Die Rotgrünen: SAP = Arbetarepartiet-Socialdemokraterna (Sozialdemokratische Partei), } \\
\text { V = Vänsterpartiet (Linkspartei), MP = Miljöpartiet de gröna (Grüne). Keinem Block zugehörig: SD = } \\
\text { Sverigedemokraterna (Schwedendemokraten). Die stärksten Parteien, die nicht den Einzug in den Reichs- } \\
\text { tag erreichten: FI = Feministiskt initiativ (Feministische Initiative), PP = Piratpartiet (Piratenpartei). } \\
\text { Quellen: Valmyndigheten (Schwedisches Wahlbehörde), Amtliches Endergebnis, http://www.val.se/val/ } \\
\text { val2010/slutresultat/protokoll/protokoll_00R.pdf (Abruf am 10. November 2010). }\end{array}$} \\
\hline
\end{tabular}

legen konnte und mit 30,1 Prozent der Stimmen nur noch kapp hinter der SAP (30,7 Prozent) liegt, steigerte die Grüne Partei ihr Wahlergebnis um 2,1 auf nun 7,3 Prozent. Alle anderen Parteien büßten in der Wählergunst an Rückhalt ein. Besonders schmerzlich waren die Verluste für die SAP, die nochmals 4,3 Prozentpunkte einbüßte; für die SAP war es eine erneute „Katastrophenwahl“5. Die geringen Verluste der Zentrumspartei führten dazu, dass die Liberale Volkspartei jetzt zweitstärkste bürgerliche Kraft ist und den Vize-Regierungschef stellt. ${ }^{6}$ Die Feministische Initiative sowie die Piratenpartei scheiterten deutlich an der Vier-Prozent-Hürde.

5 Bereits die Reichstagswahl 2006 wurde für die SAP mit den damals deutlichen Verlusten als „Katastrophenwahl“ bezeichnet, vgl. hierzu Florian Pfeil, Regierungswechsel in der Hochburg der Sozialdemokratie: Die Wahlen zum Schwedischen Reichstag vom 17. September 2006, in: ZParl 37. Jg. (2006), H. 4, S. 763 - 777; Sven Jochem, Die Reichstagswahl 2006. Eine Zäsur in der schwedischen Parteiengeschichte, in: Nordeuropaforum 2/2006, S. 5 - 24.

6 Die Regierung Reinfeldt II wurde personell nur unwesentlich verändert. Das Kabinett umfasst 24 Sitze, wovon 13 auf die Konservative Partei, vier auf die Zentrumspartei, vier auf die Liberale Volkspartei sowie drei auf die Christdemokraten entfallen. Vgl. Schwedische Regierung, http:// www.regeringen.se/sb/d/113 (Abruf am 20. Oktober 2010); sowie Dagens Nyheter, Reinfeldts nya regering, http://www.dn.se/nyheter/valet-2010/reinfeldts-nya-regering (Abruf am 5. Oktober 2010). 


\section{Parteienwettbewerb, Reformpolitik und bürgerliches Krisenmanagement während der globalen Finanzkrise}

Seit 2003 verändern sich die Konturen des schwedischen Parteienwettbewerbs. Grundlegend hierfür ist der programmatische Wandel der Konservativen Partei. Der im Oktober 2003 neu ins Amt gewählte Vorsitzende Fredrik Reinfeldt veränderte das Image seiner Partei weg von einer rigorosen Steuersenkungspartei hin zu einer „neuen Arbeiterpartei“: Zentrales Ziel „neuer" bürgerlicher Politik ist es, alle Menschen in den Arbeitsmarkt zu integrieren und ihre Abhängigkeit vom Sozialstaat zu reduzieren. Auf genuin sozialdemokratischen Politikfeldern wie Wohlfahrt oder Beschäftigung konkurriert die Partei erfolgreich mit der SAP, wobei die vermeintliche Allmacht des schwedischen Sozialstaates von der Allianz als Bürde angesehen wird. Die Konservative Partei bezeichnet sich in Anlehnung an New Labour als „Neue Konservative Partei“. Der Parteivorsitzende Reinfeldt intensivierte erfolgreich die programmatische Zusammenarbeit zwischen Konservativer Partei und den anderen Parteien des bürgerlichen Lagers. Damit konnten die historisch tiefen Gräben zwischen den bürgerlichen Parteien im Reichstag rechtzeitig vor Beginn des Wahlkampfs 2006 überwunden werden. Die vier bürgerlichen Parteien firmierten in ihrem Wahlbündnis als „Allianz für Schweden“, heute verwenden sie lediglich den Sammelbegriff einer „Allianz“.

Die zwölf Jahre andauernde Regierungsphase der SAP in Minderheitsposition endete 2006 mit einer für die Partei schmerzhaften Wahlniederlage. Noch in der Wahlnacht kündigte der damalige charismatische Parteivorsitzende Göran Persson seinen Rücktritt von allen Ämtern an - und bescherte seiner Partei eine Führungskrise. ${ }^{7}$ Auf dem Parteitag der SAP im März 2007 wurde schließlich Mona Sahlin zur neuen Vorsitzenden gewählt. Sie versprach einen neuen Führungsstil und eine stärkere Einbeziehung der Basis in zentralen politischen Streitfragen. Für viele Beobachter überraschend folgte sie dem Beispiel der Konservativen Partei und eruierte die Möglichkeiten einer formalisierten politischen Zusammenarbeit mit der Grünen Partei. Als sich schließlich im Herbst 2008 die beiden Parteien offiziell zu einem rot-grünen Wahlbündnis zusammenschlossen, geriet die SAP aus den eigenen Reihen unter Druck, auch die Linkspartei in diese Zusammenarbeit zu integrieren. ${ }^{8}$ Die Parteiführung sah viele Forderungen der Linkspartei insbesondere in den Bereichen der Haushalts- und Außenpolitik als nicht mit den Standpunkten der SAP vereinbar an. Gleichwohl beugte sie sich dem Druck der Mitglieder und insbesondere einiger LO-Arbeitergewerkschaften (Landesorganisationen i Sverige) und erweiterte das rot-grüne Wahl-

7 Die bereits in der SAP intern vorgesehene Nachfolgerin von Göran Persson, die sozialdemokratische Außenministerin Anna Lindh, fiel am 10. September 2003 einem tödlichen Attentat in der Stockholmer Innenstadt zum Opfer.

8 Die SAP unter Mona Sahlin versuchte seit 2007, die Kontakte zu den beiden anderen Parteien des linken Lagers zu intensivieren. Auf einem Treffen in Bommersvik im Juni 2008 wurde erstmals über konkrete gemeinsame Projekte verhandelt. Damals schloss die Linkspartei viele Themen der Wirtschafts- und Finanzpolitik für sie als nicht tragbar aus. Am 8. Oktober wurde die Zusammenarbeit zwischen SAP und Grüner Partei offiziell beschlossen. Nach mehreren gescheiterten Versuchen einer engeren Zusammenarbeit wurde erst am 7. Dezember 2008 offiziell das rot-grüne Bündnis und im Falle eines Wahlsieges eine rot-grüne Koalition vereinbart. Insgesamt sechs Arbeitsgruppen sollten ein gemeinsames Wahlmanifest erarbeiten. Vgl. Miljöpartiet, De rödgröna är här, http://www.mp.se/templates/Mct_177.aspx?number=161161 (Abruf am 10. November 2010). 
bündnis um die Linkspartei mit ihrem pazifistischen und kapitalismuskritischen Vorsitzenden Lars Ohly. ${ }^{\text {? }}$

Oberste Priorität der Allianz hatte in der vergangenen Wahlperiode die Bekämpfung der als hoch eingestuften Krankenstände in der schwedischen Arbeitnehmerschaft. Unmittelbar nach Regierungsantritt wurden Möglichkeiten sondiert, die Praxis der Krankschreibungen und Frühpensionierungen zu verändern. Beginnend mit dem 1. Juli 2008 traten schrittweise Reformen in Kraft, mit denen die Regeln der gesetzlichen Krankenversicherung neu formuliert wurden. Die vielfältigen Maßnahmen beinhalten eine Absenkung des Krankengeldes sowie verstärkte Ansprüche gegenüber den krankgeschriebenen Personen, wieder Arbeit - und sei es auch auf Teilzeitbasis - anzunehmen. Vor allem in der Schlussphase des Wahlkampfes berichteten die Medien von Fällen, in denen schwerkranke Menschen von der Krankenversicherung an die Arbeitsmarktbehörde verwiesen wurden. Die sozialdemokratische Spitzenkandidatin Sahlin bezeichnete die reformierte schwedische Krankenversicherung als eine der „härtesten in der westlichen Welt" ${ }^{10}$. Das rot-grüne Wahlbündnis stellte in Aussicht, diese Reformen nach gewonnener Wahl wieder rückgängig zu machen. Unmittelbar nach der Wahl bestätigte der konservative Sozialversicherungsminister Ulf Kristersson, dass einzelne Regeln der neuen Krankenversicherung überprüft, die Eckpunkte der Reform aber nicht angetastet würden. Im Gegenteil verwies der Minister auf die bisherigen Erfolge: die Anzahl der Krankschreibungen sei erstmals seit mehreren Dekaden merklich gesunken, die regionalen Differenzen nahezu verschwunden und die Anzahl der Frühpensionäre um circa 90.000 zurückgegangen. ${ }^{11}$

Die Reform der Arbeitslosenversicherung war ebenfalls zwischen den beiden politischen Lagern höchst umstritten. Die Regierung senkte die staatliche Finanzierung der in Schweden freiwilligen Arbeitslosenversicherungen, erhöhte sehr deutlich die Arbeitnehmerbeiträge und senkte die Lohnersatzleistungen ab. Da in Schweden die von den Gewerkschaften verwalteten Versicherungen freiwillig sind, reagierten viele Erwerbstätige und kündigten ihre Arbeitslosenversicherung - und zugleich ihre Mitgliedschaft in der Gewerkschaft. Seit 2007 sinkt der gewerkschaftliche Organisationsgrad rapide. ${ }^{12}$ Seitens der Opposition wird diese Politik als ein Angriff auf die gewerkschaftlichen Machtgrundlagen interpretiert. Kurz vor der Wahl wurde erneut von der Regierung überprüft, ob die Arbeitslosenversicherungen verstaatlicht werden sollten, was einer weitreichenden Entmachtung der schwedischen Gewerkschaften gleichkäme. Ministerpräsident Fredrik Reinfeldt wies jedoch darauf hin, dass eine solche Reform nicht anstehe, es sollten lediglich unterschiedliche Reformoptionen überprüft werden - Politiker der Liberalen Volkspartei hingegen fordern einen solchen Schritt. ${ }^{13}$

9 Der alte und neue Außenminister Schwedens, Carl Bildt, warnte im Wahlkampf vor den außenpolitischen Forderungen der Linkspartei und warf Lars Ohly direkt Anti-Amerikanismus vor; Carl Bildt, Sahlin har gett vika för Ohlys anti-amerikanism, in: Dagens Nyheter vom 2. Juli 2010.

10 Camilla Sundell / Anders Munk, Mona Sahlin: Vi har västvärldens tuffaste sjukförsäkring, in: Aftonbladet vom 17. September 2010.

11 Vgl. Ulf Kristersson, Alltför sjuka överförs till Arbetsförmedlingen, in: Dagens Nyheter vom 11. November 2010.

12 Vgl. Anders Kjellberg, Kollektivavtalens täckningsgrad samt organisationsgraden hos arbetsgivarfürbund och fackförbund, in: Studies in Social Policy, Industrial Relations, Working Life and Mobility, Research Reports 1/2010, Lund University; Sven Jochem, Wandel und Zukunftsaussichten des schwedisch-sozialdemokratischen Modells, in: Leviathan, 38. Jg. (2010), H. 2, S. 227 - 249.

13 Vgl. Mats J. Larsson, Oenigheit om a-kassan, in: Dagens Nyheter vom 6. Juli 2010. 
Rasch - mit Gültigkeit ab dem 1. Januar 2007 - führte die Allianz eine Form des „earned income tax credits" („Jobbskatteavdrag") ein. Schrittweise wurden die steuerlichen Belastungen insbesondere für Geringverdiener abgesenkt. Damit folgte die Allianz einem Wahlversprechen gradueller Steuersenkungen. Während der Finanzkrise wurden diese Steuererleichterungen nochmals ausgeweitet, und die bürgerliche Allianz senkte die Mehrwertsteuer für haushaltsbezogene Dienstleistungen („Rut-“ beziehungsweise „Rot-Avdrag“). Die Käufer solcher Dienstleistungen können die Abzüge (Hälfte der Mehrwertsteuer bis zu einer Grenze von umgerechnet circa $5.000 €$ pro Steuerzahler und Jahr) direkt bei der Rechnung geltend machen, wohingegen der Anbieter ein Kompensationsverfahren bei der Steuerbehörde einleiten muss. Seither nehmen die haushaltsbezogenen Dienstleistungen im Handwerk, aber auch in für den schwedischen Arbeitsmarkt untypischen Bereichen wie bei der Kinderbetreuung oder der Reinigung von Privathaushalten rasant zu. ${ }^{14}$

Die Steuerpolitik war zentrales Wahlkampfthema. Die rot-grüne Opposition kritisierte scharf, dass die Allianz Steuersenkungen durchführe, die die Balance der öffentlichen Haushalte sowie die Qualität des Sozialstaates unterminierten. Zugleich seien trotz verbaler Bekundungen und trotz der Einführung des "Jobskatteavdrages" die Entlastungseffekte für die oberen Einkommensschichten deutlich größer als für die unteren. Zum einen nutzen die oberen Einkommensschichten überdurchschnittlich oft die Möglichkeit der Mehrwertsteuerabsenkung für haushaltsbezogene Dienstleistungen. ${ }^{15}$ Zum anderen sind die Absenkungen der Einkommenssteuer für die höheren Einkommensgruppen vom Volumen her beträchtlich. Schätzungen gehen von circa zehn Milliarden Euro Steuersenkungen seit 2007 aus. Neben gesenkten Einkommenssteuern entfiel die Vermögenssteuer zur Gänze. ${ }^{16}$ Noch vor der Wahl publizierte die Regierung eine Verteilungsanalyse, in der sie als Effekt ihrer Politik eine steigende Einkommensungleichheit einräumte; dies sei jedoch ein Trend, der schon seit einer Dekade zu beobachten sei. Zudem könne auf der Habenseite eine zunehmende Integration der schwedischen Bevölkerung in den Arbeitsmarkt attestiert werden. ${ }^{17}$

Ein weiterer Bereich der Reformpolitik umfasst die forcierte Privatisierung staatlicher Betriebe. Bereits seit den 1990er Jahren haben sozialdemokratische Regierungen die ehemals staatlichen Monopole schrittweise dereguliert und teilweise auch privatisiert. ${ }^{18}$ Die bürgerliche Allianz machte von Beginn an deutlich, dass sie die Stellung des Staates im Wirtschaftsleben vehement einschränken wolle. Neben dem raschen Verkauf staatlicher Betriebe mit hohem öffentlichen Erkennungswert - wie zum Beispiel vin\&sprit, dem Hersteller des Absolut Vodkas - privatisierte die Regierung zahlreiche andere Betriebe in verschiedenen Sektoren wie Telekommunikation, Finanzwirtschaft und Immobilienhandel. Besondere Aufmerksamkeit erlangte die Privatisierung des schwedischen Apothekensystems. Im Jahr 2008 betrugen die schwedischen Privatisierungen nahezu 50 Prozent aller

14 Vgl. Tove Nandorf, Fler passar barnen med rut, in: Dagens Nyheter vom 20. Juli 2010.

15 Vgl. Lovisa Shöld, Hushållsnäre tjänster - mest för höginkomsttagare, in: Välfärd 2/2009, S. 24 -25 .

16 Vgl. Thomas Östros, Ett misslyckande ska kallas et misslyckande, in: Dagens Nyheter vom 14. September 2010.

17 Vgl. Prop. 2009/10:1, Bilaga 4 (Beilage zum Herbstbudget 2009), http://www.regeringen.se/content/1/c6/13/17/16/c795879b.pdf (Abruf am 14. November 2010).

18 Vgl. Michael Baggesen Klitgaard, Why are they doing it? Social Democracy and Market-Oriented Welfare State Reforms, in: West European Politics, 30. Jg. (2007), H. 1, S. 172 - 194. 
Privatisierungen innerhalb der EU. ${ }^{19}$ Erst mit Ausbruch der globalen Finanzkrise wurden diese Bemühungen der Regierung aufgrund der sich rapide verschlechternden Marktbedingungen gedrosselt, wenngleich an ihrer späteren Umsetzung kein Zweifel gelassen wird.

Die Reformpolitik der neuen Regierung eröffnete der Opposition zahlreiche Ansatzpunkte für Kritik. Sie kreidete der bürgerlichen Allianz an, dass sie trotz rhetorischer Bemühungen einer „neuen Arbeiterpartei“ alles tue, um das schwedische Modell in seinen Grundfesten zu erschüttern. In den Umfragen sanken die Werte für die bürgerliche Allianz rapide. Zu Beginn des Jahres 2008 lagen die rot-grünen Oppositionsparteien mit ungefähr 20 Punkten Differenz vor der bürgerlichen Allianz. Die SAP erreichte wieder klassische Umfragewerte von über 45 Prozent der Wahlberechtigten. Dieser fulminante Vorsprung der Oppositionsparteien dauerte mit Schwankungen das gesamte Jahr 2008 an, sank jedoch mit Beginn des Jahres 2009 stetig. ${ }^{20}$

Seit Ende 2008 verlor die rot-grüne Opposition ihren Kampf um die Macht. Zwei Ereignisse sind hierfür ursächlich: Zum einen verlief die Zusammenarbeit im rot-grünen Wahlbündnis keineswegs reibungslos. Zum anderen konnte die bürgerliche Allianz - und insbesondere der konservative Finanzminister Anders Borg - die globale Finanzkrise für ein die Wählerschaft offensichtlich überzeugendes Krisenmanagement nutzen.

Das im Herbst 2008 gegründete rot-grüne Wahlbündnis setzte sich zum Ziel, rechtzeitig vor der heißen Phase des Wahlkampfes in allen zentralen politischen Bereichen gemeinsame Wahlmanifeste vorlegen zu können. Während zwischen der Grünen Partei und der SAP-Elite programmatisch große Übereinstimmungen existierten, waren die Deckungsgrade mit der Linkspartei eher gering. Insofern ist es nicht überraschend, dass sich die Veröffentlichungen der rot-grünen Wahlplattformen in die Länge zogen. Die ambitionierten Zeitpläne konnten selten eingehalten werden. So wurde zum Beispiel im Februar 2010 für den Frühling ein gemeinsames Positionspapier zur rot-grünen Europapolitik angekündigt, das allerdings erst Ende Juni der Öffentlichkeit vorgestellt werden konnte. ${ }^{21}$

Die Mitglieder der SAP waren hinsichtlich der rot-grünen Zusammenarbeit gespalten. Während die klassischen Sympathisanten der Arbeitergewerkschaften einer Annäherung von sozialdemokratischer Elite und Grüner Partei aufgrund des industriekritischen Tenors der Grünen Partei eher skeptisch gegenüber stehen, sind insbesondere diejenigen Anhänger der SAP, die im öffentlichen Dienst oder in den industriefernen Dienstleistungen beschäftigt sind, eher ablehnend gegenüber den Forderungen der Linkspartei eingestellt. Viele der Antipathien gegenüber der Linkspartei entluden sich zudem an der schillernden Persönlichkeit ihres Vorsitzenden Lars Ohly. Meinungsumfragen zufolge bewegte allein seine Person jeden dritten Wähler der SAP dazu, das politische Lager zu wechseln. ${ }^{22}$

19 Vgl. OECD, Economic Survey: Sweden, Paris 2008, S. 127.

20 Die Angaben beziehen sich auf Meinungsumfragen des Forschungsinstitutes synovate, http:// www.temo.se/Templates/Page_195.aspx (Abruf am 10. November 2010). Dort sind ebenfalls die Meinungsumfragen anderer Forschungsinstitute aufgelistet.

21 Vgl. Henrik Brors, Rödgröna enade om EU-politiken, in: Dagens Nyheter vom 27. Juni 2010; Lillemor Idling, Lång väg till einighet för rödgröna, in: Dagens Nyheter vom 3. Januar 2010. Eine Liste rot-grüner Übereinkommen ist abrufbar unter http://www.socialdemokraterna.se/Var-politik/Motioner-och-rapporter/ (Abruf am 10. November 2010).

22 Vgl. Carl Melin, Var tredje S-väljare bytte block på grund av Ohly, in: Dagens Nyhter vom 21. September 2010. Carl Melin ist Geschäftsführer des Meinungsforschungsinstitutes United Minds (http://www.unitedminds.se). 
Die globale Finanzkrise traf Schweden ähnlich hart wie Deutschland. Die schwedische Finanzwirtschaft besitzt überschaubare Verflechtungen mit dem US-amerikanischen Markt, allerdings sind die Verbindungen zu den osteuropäischen und insbesondere baltischen Finanzmärkten sehr eng. Aus dieser Quelle wurden die schwedischen Finanzinstitute, denen eine allgemein gute Finanzlage zu Beginn der Krise bescheinigt wurde, negativ beeinflusst. Als stark vom Export abhängige Nation wurde Schweden nach der finanzwirtschaftlichen Krise dann auch rasch vom Einbruch der globalen Realwirtschaft getroffen. ${ }^{23}$

Die bürgerliche Allianz und insbesondere der konservative Finanzminister Anders Borg konnten diese Situation nutzen, um ihre Fähigkeiten beim ökonomischen Krisenmanagement unter Beweis zu stellen. Nach dem Ende des Zweiten Weltkrieges regierten bürgerliche Parteien stets in Zeiten ökonomischer Turbulenzen - und in der Vergangenheit bestachen sie keineswegs durch wirtschafts- oder haushaltspolitische Brillanz. Dies änderte sich im Zuge der globalen Finanzkrise nach 2008. Die Allianz reagierte anfangs eher zögerlich, allerdings ab Herbst 2008 mit Nachdruck. Nach kompromisslosen Rettungsaktionen für die schwedische Finanzwirtschaft wurden mehrere konjunkturpolitische Maßnahmen in das Herbstbudget 2008 sowie das Frühjahrsbudget 2009 integriert. ${ }^{24}$ Neben den bereits erwähnten steuerpolitischen Erleichterungen transferierte die Regierung beträchtliche Geldmittel an die Kommunen, die ohne Zielvorgaben über das Geld befinden können. Da die Kommunen in Schweden die Hauptträger des Sozialstaates sind, sollte mit dieser Maßnahme die Beschäftigungslage in den staatlichen Sozialdienstleistungen stabilisiert werden. Zudem folgte die Allianz den Pfaden klassisch sozialdemokratischer Krisenpolitik: Eine direkte Subvention einzelner Wirtschaftszweige wurde vermieden ${ }^{25}$, gleichzeitig setzte die Allianz auf Maßnahmen der aktiven Arbeitsmarktpolitik sowie einer weiter forcierten Bildungspolitik.

Als letzte historische Zäsur hob sie kurz vor Beginn der heißen Phase des Wahlkampfes den Atomausstieg auf. Nach einer langen und hitzig geführten Plenardebatte im Juni 2010 wurde das Ergebnis der konsultativen Volksabstimmung aus dem Jahre 1980 revidiert. Neue Kernkraftwerke können in Schweden gebaut werden, allerdings nur an bereits bestehenden Standorten und als Ersatz für ältere Reaktoren. Dennoch ist mit diesem Entscheid des Parlaments die weitere Nutzung der Kernenergie Teil der schwedischen Energiepolitik. Dabei konnte die Allianz ihren Antrag mit nur zwei Stimmen Mehrheit durchbringen. Aus der agrarisch geprägten Zentrumspartei stammten die Reichstagsmitglieder, die sich gegen die Linie ihrer eigenen Fraktion beziehungsweise ihres eigenen Wahlbündnisses stellten.

23 Vgl. hierzu und zu den folgenden Ausführungen: Sven Jochem, Sweden Country Report, in: Bertelsmann Stiftung (Hrsg.), Managing the Crisis. A Comparative Assessment of Economic Governance in 14 Economies, Gütersloh 2010 (mit weiteren Literaturverweisen).

24 In Schweden ist der Budgetprozess so gegliedert, dass im Frühlingsbudget die großen Richtlinien der Haushaltspolitik für das kommende Jahr beschlossen werden. Im Herbstbudget bestehen hingegen Spielräume für Feinjustierungen und auch weitere Schwerpunktsetzungen. Vgl. http:// www.regeringen.se/sb/d/2459 (Abruf am 10. November 2010). Vgl. zur Finanzpolitik insgesamt die Evaluationen des unabhängigen Expertengremiums „Finanspolitiska Rådet“ unter dem Vorsitz von Lars Calmfors, http://www.finanspolitiskaradet.se (Abruf am 23. November 2010).

25 Diese Politik war seitens der Opposition und der Gewerkschaften vor allem im Falle der schwedischen Automobilindustrie heftig umstritten. Diese Akteure forderten eine Politik wie in Deutschland zur Stärkung von Automobilherstellern wie Volvo oder Scania sowie deren Zulieferern. 
Für die Zentrumspartei, die sich auch als ökologische Partei der dünn besiedelten Regionen Schwedens sieht, stellte diese Entscheidung eine schwere Hypothek für den Wahlkampf dar.

Die heiße Phase des Wahlkampfes zeichnete sich durch Skandale in der Konservativen Partei sowie eine heftige Auseinandersetzung um die sozialdemokratische Spitzenkandidatin Sahlin aus. Im Sommer 2010 trat der konservative Arbeitsminister Sven Otto Littorin zurück. Er war aufgrund seiner Reformen der Arbeitsmarktpolitik in der Bevölkerung umstritten. Allerdings kommunizierte er offen und mutig Schwierigkeiten im Zusammenhang mit seiner Scheidung sowie Gesundheitsprobleme, was ihm gewisse Sympathien in der Öffentlichkeit einbrachte. Grund des Rücktrittes waren Anschuldigungen des der Sozialdemokratie nahe stehenden Boulevardblattes Aftonbladet. Darin wurde dem Minister vorgeworfen, Sex gekauft zu haben - in Schweden ist dies eine Straftat. Der Minister trat unmittelbar nach den ersten Vorwürfen zurück mit Verweis darauf, dass er sich und seine Kinder vor der Öffentlichkeit schützen wolle. ${ }^{26}$

Der Wahlkampf in Schweden ist verstärkt auf Persönlichkeiten ausgerichtet. Mit der Blocklogik des schwedischen Parteienwettbewerbs rückten die Spitzenkandidaten der jeweiligen Lager immer stärker ins mediale Rampenlicht. Während sich Fredrik Reinfeldt 2006 noch als unverbrauchter Konkurrent gegen den ermattenden Göran Persson in der Öffentlichkeit in Szene setzen konnte, war sein Rückhalt in der Öffentlichkeit bei der Wahl 2010 geringer. Allerdings litt die SAP unter der schlechten Berichterstattung über ihre Spitzenkandidatin Mona Sahlin. Insbesondere die bürgerliche Boulevardzeitung Expressen publizierte 75 Prozent ihrer Berichterstattung über Sahlin mit deutlich negativer Wertung. ${ }^{27}$ Zudem wurde diese im Frühsommer 2010 Ziel der Kritik aufgrund ihrer so genannten „Toblerone-Affäre“: Sie hatte 1995 als Ministerin für Gleichstellungsaufgaben und stellvertretende Ministerpräsidentin private Dinge, darunter auch Süßigkeiten, mit der Dienstkreditkarte gekauft. Sie konnte zwar seinerzeit nachweisen, dass sie die Beträge stets ausgeglichen hatte, allerdings war der öffentliche Druck so groß, dass sie von ihren politischen Ämtern zurücktrat und 1996 den Reichstag verließ. Als die rot-grünen Parteien ihren Budgetentwurf präsentierten, kritisierte ihn der christdemokratische Kommunalminister Mats Odell mit dem Hinweis, dies sei reine „Toblerone-Politik“, man kaufe Dinge mit einer Karte und präsentiere die Rechnung dann anschließend dem schwedischen Volk. ${ }^{28}$ Fast alle führenden Repräsentanten der SAP wiesen diese Anschuldigungen mit Nachdruck zurück und stellten sich geschlossen hinter die Spitzenkandidatin. ${ }^{29}$

26 Vgl. Cecilia Jacobsson, Littorin: Snudd på outhärdligt, in: Dagens Nyheter vom 14. Juli 2010; Niklas Ekdal, Littorinskandalen en katastrof för vårt politiska system, in: Dagens Nyheter vom 13. Juli 2010.

27 Vgl. Kent Asp, Starkt negativ rapportering om Mona Sahlin i Expressen, in: Dagens Nyheter vom 20. September 2010.

28 Vgl. Kalle Holmberg, KD-tal om Toblerone fick rödgröna at ilsna till, in: Dagens Nyheter vom 27. April 2010.

29 Vgl. Ann-Kristine Johansson, Stoppa hatkampanjen mot Mona Sahlin, in: Dagens Nyheter vom 3. Juli 2010; Ingvar Carlsson u.a., Vi tar strid för vår partiordförande, in: Dagens Nyheter vom 14. September 2010. 


\begin{tabular}{|c|c|c|c|c|c|c|c|c|c|c|c|c|}
\hline \multirow[b]{2}{*}{ Wahl 2006} & \multicolumn{11}{|c|}{ Wahl 2010} & \multirow[b]{2}{*}{$\Sigma$} \\
\hline & V & SAP & MP & C & FP & KD & M & SD & FI & PP & Andere & \\
\hline $\mathrm{V}$ & 62 & 19 & 10 & 1 & 1 & 1 & 2 & 1 & 2 & 0 & 0 & -100 \\
\hline SAP & 4 & 73 & 7 & 1 & 2 & 1 & 8 & 3 & 0 & 0 & 0 & -100 \\
\hline MP & 11 & 10 & 62 & 3 & 2 & 1 & 5 & 1 & 3 & 1 & 0 & -100 \\
\hline $\mathrm{C}$ & 2 & 7 & 7 & 56 & 9 & 4 & 14 & 1 & 0 & 1 & 0 & -100 \\
\hline FP & 1 & 5 & 5 & 11 & 49 & 5 & 20 & 2 & 1 & 1 & 0 & -100 \\
\hline $\mathrm{KD}$ & 1 & 8 & 3 & 5 & 6 & 60 & 14 & 2 & 0 & 0 & 0 & -100 \\
\hline M & 0 & 5 & 1 & 6 & 6 & 5 & 73 & 3 & 0 & 0 & 0 & -100 \\
\hline SD & 0 & 17 & 1 & 0 & 4 & 1 & 8 & 66 & 0 & 0 & 2 & -100 \\
\hline Andere & 18 & 16 & 11 & 3 & 7 & 2 & 11 & 7 & 8 & 12 & 3 & -100 \\
\hline Ungültig & 9 & 24 & 11 & 6 & 7 & 3 & 27 & 9 & 2 & 2 & 1 & -100 \\
\hline Nichtwahl & 7 & 34 & 11 & 6 & 3 & 3 & 28 & 6 & 0 & 1 & 1 & -100 \\
\hline $\begin{array}{l}\text { Nicht wahl- } \\
\text { berechtigt }\end{array}$ & 9 & 28 & 17 & 5 & 7 & 3 & 22 & 6 & 1 & 2 & 0 & -100 \\
\hline
\end{tabular}

\section{Die Wablentscheidung}

Die rot-grüne Opposition büßte ihren sicheren Vorsprung in den Meinungsumfragen seit Herbst 2008 kontinuierlich ein. Für die Wähler waren vor allem Fragen der Bildungs-, Beschäftigungs- sowie Wirtschaftspolitik entscheidend - Themen, die eher traditionell von der Sozialdemokratie besetzt waren. Weniger als ein Drittel der Wähler machte die umstrittene Frage der Kernkraft für die Entscheidung verantwortlich, und nur 26 Prozent sahen die Einwanderungsfrage als relevant an, wenngleich dies für die Wähler der Schwedendemokraten ein wichtiges Thema des Wahlkampfes war. ${ }^{30}$

Die Daten zur Wählerwanderung zeigen, dass 12 Prozent der SAP-Wähler zu Parteien der bürgerlichen Allianz, insbesondere der Konservativen Partei, wechselten (vgl. Tabelle 2). Die rechtpopulistischen Schwedendemokraten bekamen Unterstützung zu gleichen Teilen von ehemaligen Anhängern der SAP und der Konservativen Partei. Ein besonders großer Anteil der SD-Wählerschaft wurde von Wählern der Parteien mobilisiert, die 2006 nicht den Sprung in den Reichstag geschafft hatten. Bei den Erstwählern kam die SD auf einen Anteil, der ungefähr dem allgemeinen Wahlergebnis entspricht. Bei den geringen Machtverschiebungen entschieden die Wechselwähler durch taktisches Wählen den Ausgang. ${ }^{31}$

Das schlechte Abschneiden der Sozialdemokraten kann auf Verluste nach links wie nach rechts zurückgeführt werden. Obwohl die SAP (gleichauf mit den Konservativen) den

30 Vgl. Sören Holmberg / Per Näsman / Kent Wänström, Riksdagsvalet 2010. Valu, Sveriges Television, S. 8, http://svt.se/content/1/c8/02/15/63/14/ValuResultat2010_100921.pdf (Abruf am 20. Oktober 2010).

31 Vgl. Mikael Gilljam, Taktikröstare använder sin makt, in: Svenska Dagbladet vom 16. September 2010. 
höchsten Anteil „treuer“ Wähler in der schwedischen Parteienlandschaft hat, wanderten erhebliche Anteile sowohl zur Linkspartei als auch zu den Grünen. Ebenfalls bemerkenswert ist die Wanderung von insgesamt acht Prozent ehemaliger SAP-Wähler allein zur Konservativen Partei. Dieser Wechsel ist exakt so groß wie bei der Reichstagswahl 2006 und zeugt von stetiger Abwanderung sozialdemokratischer Wähler hin zur „,neuen Arbeiterpartei“, den Konservativen. ${ }^{32}$

Die soziale Lage der Wähler bestimmt noch immer zu einem großen Teil die Wahlentscheidung. ${ }^{33}$ Allerdings ist bemerkenswert, dass von allen erwerbstätigen Personen 32 Prozent die Konservative Partei wählten, hingegen nur 22 Prozent die SAP. Deutliche Mehrheiten ergaben sich für die SAP bei den Arbeitslosen, Krankgeschriebenen und Frühpensionären. Es scheint, dass die Sozialdemokraten gerade bei jenen Bevölkerungsgruppen großen Rückhalt erfahren, die Hauptziel bürgerlicher Reformpolitik sind. Die Mehrzahl der Anhänger der klassischen Arbeitergewerkschaften (LO) wählte noch die SAP (51 Prozent), allerdings entschieden sich bereits 16 Prozent von ihnen für die Konservativen. In diesem Wählersegment konnte die Konservative Partei im Vergleich zu 2006 um knapp vier Prozentpunkte zulegen. Während die Anhängerschaft der Angestelltengewerkschaften (TCO) nahezu gleich stark die SAP (26 Prozent) oder die Konservative Partei (29 Prozent) wählte, votierten lediglich 18 Prozent der Anhänger der Akademikergewerkschaften für die SAP, hingegen 28 Prozent für die Konservative Partei. Die Mehrheit der über 65 Jahre alten Wahlbürger entschied sich für die SAP (34 Prozent), wobei dieser Wert seit der Wahl von 2002 stark rückläufig ist (von 44 Prozent). Entsprechend konnte die Konservative Partei in dieser Wählergruppe deutlich von 15 (2002) auf 28 Prozent (2010) zulegen. Bei den Wählern, die im Ausland geboren wurden, führt die SAP hingegen deutlich mit 49 Prozent der Stimmen gegenüber lediglich 20 Prozent für die Konservative Partei.

Eine gewichtige Rolle bei der Wahlentscheidung kam den Spitzenkandidaten zu. In Schweden ist die Orientierung auf das Spitzenpersonal für gewöhnlich nicht stark ausgeprägt. Allerdings hat sich dies seit der Wahl von 2006 und der sich durchsetzenden Blocklogik des Parteienwettbewerbs deutlich geändert. Die Spitzenkandidaten Reinfeldt und Sablin standen für klare politische Alternativen. Aufgrund des erfolgreichen Krisenmanagements überrascht es nicht, dass in fast allen Punkten der Konservative Reinfeldt deutlich vor seiner sozialdemokratischen Herausforderin in der Wählergunst wahrgenommen wurde. Vor allem das internationale Auftreten, die ökonomische Kompetenz und die Führungskraft wurden bei Reinfeldt höher eingeschätzt als bei Sahlin. ${ }^{34}$

Der Bruch in der schwedischen Politik wird deutlich, wenn die Kompetenzzuweisungen der Bürger nach bestimmten Sachfragen analysiert werden. Bei den Themen Ökonomie, Beschäftigung, Bildung und Steuern sahen zum Teil deutlich mehr als 50 Prozent der Wahlberechtigten die Parteien der Allianz als kompetenter an. Dies waren ehemals Politikfelder, in denen die Sozialdemokratie dominierte. Das rot-grüne Lager konnte lediglich in den Bereichen Umweltschutz, Gesundheitswesen und Sozialpolitik die Mehrheit der Wähler von ihren Kompetenzen überzeugen.

32 Vgl. Sven Jochem, a.a.O. (Fn. 5), S. 16.

33 Die folgenden Ausführungen basieren auf Sören Holmberg / Per Näsman / Kent Wänström, a.a.O. (Fn. 30).

34 Vgl. Henrik Bors, Reinfeldt slår Sahlin på samtliga punkter, in: Dagens Nyheter vom 16. Juni 2010. 
Die Wahl 2010 zeugt von einer weiteren politischen Verschiebung hin zum rechten Parteienspektrum. Die Allianz und vor allem die Konservative Partei konnte klassisch sozialdemokratische Themenfelder mit Erfolg besetzen. Ebenso deutlich konnte die Partei in den Gruppen der Kernwähler der SAP zunehmend Unterstützung mobilisieren. Zu diesem Bild passt, dass sich immer weniger Wähler in Schweden als ,links" einstufen. Dieser Wert ging von 44 (2002) auf 38 Prozent zurück, wohingegen die Selbsteinschätzung als „rechts“ von 34 (2002) auf 42 Prozent zunahm. Die Reichstagswahl 2010 wurde nicht nur mit einer sehr hohen Wahlbeteiligung abgeschlossen (84,6 Prozent), sondern offenbarte auch, dass das Vertrauen der schwedischen Wähler in die Politiker von 54 Prozent im Jahre 2006 auf 70 Prozent angestiegen war. Ein Vertrauensanstieg ist bei Wählern aller Parteien zu verzeichnen, fiel jedoch sehr stark bei den Wählern der Allianz aus, die zu ungefähr 80 Prozent den Politikern vertrauten.

\section{Dynamiken des Parteienwettbewerbs in der schwedischen Demokratie}

Die bürgerliche Allianz unter Führung der Konservativen Partei war unmittelbar nach der Wahl bestrebt, keine weiteren großen Reformvorhaben in der Öffentlichkeit zu lancieren. Im Gegenteil verblieb der erste Budgetentwurf ${ }^{35}$ der neuen Regierung in den gewohnten Bahnen bisheriger Finanz- und Haushaltspolitik. Die wiedergewählte bürgerliche Koalition kann auf sehr gute ökonomische Rahmendaten verweisen - und interpretiert dies als Erfolg ihrer Krisenpolitik.

Gleich nach der Wahl versuchte der neue und alte Ministerpräsident Reinfeldt auch, die Grünen für eine offizielle Kooperation zu gewinnen (von ihrer Einbindung in die Koalition war nie die Rede). ${ }^{36}$ Diese wiesen das Angebot jedoch zurück. ${ }^{37}$ Ein erstes Beispiel für eine blockübergreifende Kooperation war der Beschluss zur Afghanistan-Politik. Am 1. November 2010 verständigte sich die Regierung mit der Grünen Partei sowie der SAP auf eine gemeinsame Politik, die feste Zeitpunkte für einen Rückzug der schwedischen Truppen aus Afghanistan nannte. Hierbei folgte die Regierung der Forderung der Opposition. Mit dieser Übereinkunft wird deutlich, dass die Regierung in ihrer Minderheitsposition eine pragmatische Zusammenarbeit anstrebt. Und in solch zentralen Fragen versucht sie, den parlamentarischen Rückhalt sehr breit zu gestalten, also auch die SAP einzubinden. Allerdings zeigen auch beide Oppositionsparteien, dass sie bereit sind, von ihren Maximalforderungen aus der Zeit der rot-grünen Zusammenarbeit abzurücken. ${ }^{38}$ Nach diesem Kooperationsmuster werden sowohl die Schwedendemokraten als auch die Linkspartei von zentralen politischen Entscheidungen isoliert.

35 Der Budgetentwurf ist abrufbar unter http://regeringen.se/sb/d/12677/a/153307 (Abruf am 18. November 2010).

36 In Schweden herrscht das Prinzip des negativen Parlamentarismus, nach dem eine Regierung auch nach Wahlen im Amt bleiben kann, solange ihr nicht das parlamentarische Vertrauen mit über 50 Prozent der Stimmen entzogen wird.

37 Vgl. Owe Nilsson / Peter Wallberg, Inget regeringssamarbeite med MP, in: Dagens Nyheter vom 27. Februar 2010.

38 Die Übereinkunft findet man unter http://www.regeringen.se/content/1/c6/15/48/76/aa0299ba. pdf (Abruf am 15. November 2010). Vgl. auch Dagens Nyheter, Nödvändig enighet, 3. November 2010 . 
Im rot-grünen Lager führte die Wahl zu großer Enttäuschung. Die Sozialdemokraten setzten als erste Reaktion eine Krisenkommission ein, die auf der Grundlage von umfassenden Wahlanalysen eine programmatische Neuausrichtung der Partei sowie organisatorische Reformen beraten soll. ${ }^{39}$ Kurz nach Einsetzung dieser Kommission kündigte die SAP eine Pause rot-grüner Zusammenarbeit an. ${ }^{40}$ Mona Sahlin führte aus, dass ihre Partei zuerst in einem internen Prozess die Wahlniederlage analysieren und verarbeiten müsse, bevor an weitere Kooperationen gedacht werden könne. Für viele Beobachter überraschend kündigte sie am 14. November 2010 ihren Rücktritt als Parteivorsitzende an. Hintergrund dieser Entscheidung sind parteiinterne Forderungen, insbesondere aus der Jugendorganisation (SSU), dass die gesamte Parteiführung nach dem „katastrophalen“ Wahlergebnis ihre Ämter zur Verfügung stellen solle. Die Neubesetzung des Parteivorsitzes soll vom 25. bis 27. März 2011 auf einem Sonderparteitag beschlossen werden. Allerdings zeichnet sich bislang keine eindeutig favorisierte Persönlichkeit ab. Die schwedische Sozialdemokratie hat ein Führungsproblem.

Mit dem Einzug der rechtspopulistischen Schwedendemokraten in den Reichstag folgt die schwedische Parteienlandschaft den Entwicklungen der nordischen Nachbarländer. Dort sind Rechtspopulisten seit einigen Jahren fester Bestandteil des Parteienspektrums; vor allem in Dänemark konnte sich in der Vergangenheit die Dänische Volkspartei (DF) als Unterstützungspartei bürgerlicher Minderheitskoalitionen etablieren. Die Schwedendemokraten entspringen mehreren parteilichen Fusionen und Neugründungen des rechten Lagers, von denen einzelne Quellen auch auf Gruppen aus dem militanten rechtsextremen Spektrum zurückgehen. Die Partei firmiert seit 1988 unter ihrem heutigen Namen. Der jetzige Parteivorsitzende, Per Jimmie Åkesson, ist ehemaliges Mitglied der Konservativen Partei. Er war bestrebt, in seiner Amtsperiode rechtsextreme Tendenzen in der Partei zu unterbinden. Die Programmatik der Schwedendemokraten entspricht dem rechtspopulistischen Mainstream der nordischen Länder: Aus Furcht vor „Überfremdung“ soll die Einwanderung eingeschränkt werden, sinkende Steuern sollen kombiniert werden mit steigenden sozialpolitischen Leistungen für die gebürtigen Schweden, und die europäische Integration wird rundum abgelehnt. ${ }^{41}$

Die jetzige Situation des Parteienwettbewerbs weist der Grünen Partei die Rolle als Zünglein an der Waage zu. Sie hat eine zentripetale Ausrichtung im Parteienwettbewerb und kritisiert unter anderem die am industriellen Sektor orientierte Politik der LO sowie Teile der SAP. Zudem wird auch von ihr eine Rückführung des schwedischen Staates gefordert. Insofern überrascht es nicht, dass Teile der Anhänger dieser Partei einer Zusammenar-

39 Vgl. http://www.socialdemokraterna.se/vart-parti/kriskommissionen-tillsatt/ (Abruf am 6. November 2010).

40 Vgl. Anders Bolling / Ewa Stenberg, Rödgrönt samarbete tar paus, in: Dagens Nyheter vom 26. Oktober 2010.

41 Vgl. Werner T. Bauer, Willkommen in der Normalität? Anmerkungen zum Wahlerfolg der rechten Schwedendemokraten, Internationale Politikfeldanalyse, Friedrich-Ebert-Stiftung, Berlin 2010. Bei Eröffnung des Reichstages am 2. November 2010 kam es zu einem Eklat: Die Fraktion der Schwedendemokraten verließ den traditionellen Gottesdienst unter Protest, als die Bischöfin Eva Brunne sich gegen Fremdenfeindlichkeit aussprach und forderte, dass Christen nicht Menschen aufgrund ihres Geburtslandes diskriminieren sollten. Vgl. Mikael Bondesson, SD:s ledamöter lämnade Storkyrkan, in: Dagens Nyheter vom 3. November 2010. 
beit mit der Allianz nicht abgeneigt sind. ${ }^{42}$ Die Pause in der rot-grünen Zusammenarbeit eröffnet jetzt nicht nur der Regierung vielfältige Verhandlungsoptionen, sondern eröffnet auch der Grünen Partei eine Kooperation mit der Regierung, um erfolgreich „Policy-Seeking" zu betreiben. Wie belastbar eine solche Zusammenarbeit ist, wird sich zeigen müssen.

Eine sozialdemokratische Hochburg in Europa löst sich auf. Die schwedische Sozialdemokratie folgt in ihrem Niedergang einem internationalen Trend. Dementsprechend kann die Erosion der SAP auf die Vielzahl von Einflussfaktoren zurückgeführt werden, die in vergleichenden Analysen der Sozialdemokratie diskutiert werden: die Auflösung des klassisch sozialdemokratischen Kernmilieus, die durch Grüne Parteien entstandene Konkurrenz oder die Erschöpfung des keynesianischen Sozialstaatsprojektes sowie der Aufstieg eines dominanten neo-liberalen Politikparadigmas. Auch „strategische Fehler" sozialdemokratischer Eliten verursachen den Niedergang der SAP mit. Nach Bo Rothstein liegt der Grund für die Wahlniederlage der Sozialdemokraten darin, dass sie sich von einem universellen Politikmuster entfernt und eine „postmoderne, ahistorische Identitätspolitik“ betrieben haben, in der stets nur bestimmte Randgruppen zu den Nutznießern ihrer Politik gehörten. Die SAP habe das universelle Prinzip des Wohlfahrtsstaates aufgegeben und somit die schwedische Mittelklasse verprellt. ${ }^{43}$

Die Logik der zerfallenden sozialdemokratischen Hochburg in Schweden beruht aber auch auf genuinen Bedingungen des dortigen Parteienwettbewerbs. Die SAP konkurriert mit einer neuen Konservativen Partei, die von Programmatik und Auftreten eher einer Sozialstaatspartei der Mitte ähnelt. In der Wahrnehmung von Fredrik Reinfeldt hat die Konservative Partei die SAP als „staatstragende Partei“ bereits abgelöst. ${ }^{44}$ Für die Sozialdemokratie ist die Konkurrenz mit einer Sozialstaatspartei der Mitte brisant - dies zeigen nicht zuletzt die Erfahrungen der SPD. Zudem zeigt sich, dass die bürgerliche Allianz trotz weiterhin bestehender programmatischer Differenzen diszipliniert genug ist, um wenige Angriffsflächen für die SAP zu bieten. Die Stärke der bürgerlichen Allianz ist insofern die Schwäche der SAP - und umgekehrt. Es bleibt abzuwarten, inwiefern die Allianz in Minderheitsposition auf die Grüne Partei als Unterstützerin wird bauen können, und wie es ihr gelingt, die Schwedendemokraten zu isolieren. Ebenso offen ist die Frage, ob es einer reformierten SAP gelingen kann, strategische Brücken zu den bürgerlichen Mitteparteien zu schlagen. Das rot-grüne Experiment mit Einschluss der Linkspartei ist in Schweden allerdings vorerst gescheitert.

4221 Prozent der Wähler der Grünen Partei wünschen sich explizit eine Zusammenarbeit mit der Allianz. Vgl. Sören Holmberg / Per Näsman / Kent Wänström, a.a.O. (Fn. 30), S. 29.

43 Vgl. Bo Rothstein, Därför har socialdemokratin havererat, in: newsmill.se vom 22. September 2010. Vgl. mit ähnlichen Argumenten Jonas Hinnfors, Kraftull ideologisk linje kann rädda S, in: Göteborgs-Posten vom 16. November 2010.

44 Vgl. Fredrik Reinfeldt, Moderaterna övertar rollen som samhällsbärande parti, in: Dagens Nyheter vom 13. Juli 2010. 